\title{
Relation between spin Hall effect and anomalous Hall effect in $3 d$ ferromagnetic metals
}

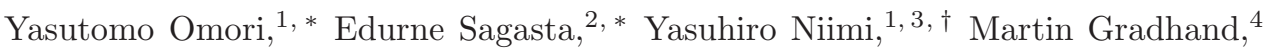 \\ Luis E. Hueso, ${ }^{2,5}$ Fèlix Casanova, ${ }^{2,5}$ and YoshiChika Otani ${ }^{1,6}$, 用 \\ ${ }^{1}$ Institute for Solid State Physics, University of Tokyo, Kashiwa, Chiba 277-8581, Japan \\ ${ }^{2}$ CIC nanoGUNE, 20018 Donostia-San Sebastian, Basque Country, Spain \\ ${ }^{3}$ Department of Physics, Graduate School of Science, \\ Osaka University, Toyonaka, Osaka 560-0043, Japan \\ ${ }^{4}$ H. H. Wills Physics Laboratory, University of Bristol, Bristol BS8 1TL, United Kingdom \\ ${ }^{5}$ IKERBASQUE, Basque Foundation for Science, 48011 Bilbao, Basque Country, Spain \\ ${ }^{6}$ RIKEN-CEMS, 2-1 Hirosawa, Wako, Saitama 351-0198, Japan
}

(Dated: January 3, 2019)

\begin{abstract}
We study the mechanisms of the spin Hall effect (SHE) and anomalous Hall effect (AHE) in $3 d$ ferromagnetic metals ( $\mathrm{Fe}, \mathrm{Co}$, permalloy $\left(\mathrm{Ni}_{81} \mathrm{Fe}_{19} ; \mathrm{Py}\right)$, and $\mathrm{Ni}$ ) by varying their resistivities and temperature. At low temperatures where the phonon scattering is negligible, the skew scattering coefficients of the SHE and AHE in Py are related to its spin polarization. However, this simple relation breaks down for Py at higher temperatures as well as for the other ferromagnetic metals at any temperature. We find that, in general, the relation between the SHE and AHE is more complex, with the temperature dependence of the SHE being much stronger than that of AHE.
\end{abstract}

PACS numbers: 72.25.Ba, 72.25.Mk, 75.70.Cn, 75.75.-c

\section{INTRODUCTION}

The spin Hall effect (SHE) and its inverse (ISHE) enable us to interconvert spin and charge currents in the transverse direction and are widely recognized as essential methods to generate and detect spin currents in spintronic devices $\underline{-1} \underline{\underline{3}}$. Since the original predictions of the SHE ${ }^{4,5}$, it has been experimentally investigated in a variety of nonmagnetic materials with strong spin-orbit interactions such as III-V semiconductors $6.7,4 d$ and $5 d$ transition metals $\stackrel{-16}{-16}$, alloys $\underline{17}-\underline{20}$, oxides $\underline{21}$, and organic materials 22 . The mechanism of the SHE can be extrinsic or intrinsic. The former depends on the combination of the host metal and impurities ${ }^{23}-25$, while the latter depends on the detailed properties of the momentum-space Berry phase $\underline{26,27}$. These mechanisms are the same as for the anomalous Hall effect (AHE) in ferromagnetic metals (FMs), which has been intensively studied for many years 28 . Thus, it has been commonly accepted that the SHE shares the same origin as the $\mathrm{AHE}^{29}$.

It was experimentally verified that not only the ISHE $^{30}-32$ but also the $\mathrm{SHE}^{33}-37$ occur in FMs with finite spin polarization. In FMs, both spin and charge accumulations can exist $\frac{38}{38}$ and are detected as the SHE and the AHE, respectively [see Fig. 1(a)]. Very recently, the control of the spin accumulation by manipulating the magnetization of the ferromagnet has been achieved experimentally ${ }^{33-36}$ after its theoretical predictiont ${ }^{38}$. Das et al .33 used the term "anomalous SHE" to describe this mechanism. The anomalous SHE can be understood by generalizing the spin conductivity $\sigma_{i j}^{s}$ to $\sigma_{i j}^{s}(M)$ allowing for the anisotropy of $\sigma_{i j}^{s}$ as discussed by Seemann et al ${ }^{39}$. Here $i j$ are the spatial directions, $s$ refers to the direction of the spin and $M$ is the global magnetization of the fer- romagnet. For the simplest contribution to this general response, in other words, $M \propto s$, it was experimentally verified that the SHE also occurs in FMs with finite spin polarization. Intuitively, in FMs, both spin and charge accumulations can exist and are detected as the SHE and the AHE, respectively [see Fig. 1(a)]. Thus, it was suggested that the SHE and AHE in FMs are related via the spin polarization 31 . However, it has not been experimentally verified if this simple relation is general, and therefore valid for all the FMs and all the mechanisms. From a theoretical viewpoint, such a relation might hold in the limit of diffusive transport $\underline{40}$ but is not expected to hold in general.

In this work, we present a detailed investigation of the relation between the SHE and the AHE in four different $3 d \mathrm{FMs}$, i.e., $\mathrm{Fe}$, Co, permalloy $\left(\mathrm{Ni}_{81} \mathrm{Fe}_{19} ; \mathrm{Py}\right)$, and Ni. By changing the residual resistivity of the FM at low temperatures, the skew scattering contribution (one of the extrinsic mechanisms) $\underline{41}$ can be separated from other contributions. It turns out that the aforementioned relation between the SHE and AHE holds for the skew scattering term in Py. However, this simple relation is not valid for the other mechanisms in $\mathrm{Py}$ and for the other FMs. The SHE in the $3 d$ FMs has much stronger temperature dependence than the AHE. We discuss a possible scenario to explain the observed results.

\section{EXPERIMENTAL DETAILS}

To perform the SHE measurements in the $3 d \mathrm{FMs}$, we adopted the spin absorption method in the lateral spin valve structure $13,15,16,18-21$. This method enables us to estimate the spin diffusion length and the spin Hall angle $\left(\theta_{\mathrm{SHE}}\right)$ on the same device. The SHE devices were 



FIG. 1: (a) Intuitive schematic of the SHE and the AHE in FM. Spin and charge accumulations appear in the transverse direction respect to the incident current $I_{\mathrm{C}}$ and are detected as the SHE and the AHE, respectively. (b) Schematic of a lateral spin valve for the spin absorption method to measure the ISHE in FM. The magnetic field $H$ is applied along the $\mathrm{Cu}$ wire. (c) Inverse spin Hall resistance $R_{\mathrm{ISHE}}$ of the middle Py wire (20 nm in thickness) as a function of $H$ at $10 \mathrm{~K}$ using the configuration shown in (b). The ISHE signal $\left(\Delta R_{\mathrm{ISHE}}\right)$ is defined in the figure. (d) Anomalous Hall resistance $R_{\mathrm{AHE}}$ of Py as a function of $H$ at $10 \mathrm{~K}$. The AHE signal $\left(\Delta R_{\mathrm{AHE}}\right)$ is defined in the figure. The inset shows a part of a Hall bar for the AHE measurement. The magnetic field $H$ is applied perpendicular to the plane. Compared to the SHE configuration, the field direction is rotated by $90^{\circ}$.

fabricated on $\mathrm{SiO}_{2} / \mathrm{Si}$ substrates with multiple-step electron beam lithography followed by metal deposition and lift-off. We first patterned two 100-nm-wide wires and deposited Py by $30 \mathrm{~nm}$ in thickness by electron beam evaporation. The two Py wires are separated by a length $(L)$ of $1 \mu \mathrm{m}$, as illustrated in Fig. 1(b). One of the Py wires is used as a spin current injector, while the other is used to estimate the spin diffusion length of our target wire, as detailed in Supplemental Material $\stackrel{42}{2}$. In the second step, the target $3 d$ FM wire (hereafter middle wire) with the width $\left(w_{\mathrm{M}}\right)$ of $200 \mathrm{~nm}$ was placed just in the middle of the two Py wires and a 5- to 30-nm-thick $3 d$ FM (Fe, Co, $\mathrm{Py}$, or $\mathrm{Ni}$ ) was deposited with electron beam evaporation. In the third step, a 100-nm-wide and 100-nm-thick $\mathrm{Cu}$ strip was bridged on top of the three wires with a Joule heating evaporator. Before the $\mathrm{Cu}$ evaporation, an Ar-ion milling treatment was performed to achieve transparent interfaces. For the AHE measurements, a $20-\mu \mathrm{m}$-long and $3-\mu \mathrm{m}$-wide Hall bar was patterned with electron beam lithography and the FM (5 to $30 \mathrm{~nm}$ in thickness) was deposited at the same time as the SHE devices were prepared. We then capped all the devices with $\mathrm{Al}_{2} \mathrm{O}_{3}$ using radio frequency magnetron sputtering to protect them from oxidization. All the electric transport measurements were performed in a ${ }^{4} \mathrm{He}$ flow cryostat using the lock-in technique.

\section{RESULTS AND DISCUSSIONS}

\section{A. SHE and AHE in Py}

When an electric current $I_{\mathrm{C}}$ is injected from Py to the left side of $\mathrm{Cu}$ as shown in Fig. T(b), spin accumulation is created at the interface and diffuses in the $\mathrm{Cu}$ bridge. In this process, a pure spin current $I_{\mathrm{S}}$ flows in the $\mathrm{Cu}$ channel on the right side. Most of $I_{\mathrm{S}}$ is then absorbed into the middle wire and converted into charge current via the ISHE, which is detected as a voltage drop $V_{\text {ISHE }}\left(=V_{+}-V_{-}\right)$. The ISHE resistance $R_{\mathrm{ISHE}}=V_{\mathrm{ISHE}} / I_{\mathrm{C}}$ is measured by sweeping the external magnetic field $H$ along the $\mathrm{Cu}$ channel. It is saturated when the magnetization of the Py wire is fully polarized. The difference of $R_{\mathrm{ISHE}}$ between the positive and negative saturated magnetic fields is the ISHE signal, defined as $2 \Delta R_{\mathrm{ISHE}}$. As shown in Fig. 1(c), a positive $\Delta R_{\mathrm{ISHE}}(\sim 25 \mu \Omega)$ was obtained at $10 \mathrm{~K}$ for a $20 \mathrm{~nm}$ thick Py middle wire with the longitudinal resistivity $\rho_{x x}$ of $22 \mu \Omega \cdot \mathrm{cm}$.

We also confirmed the reciprocity in the present system for FM. This can be realized by exchanging the electrodes $\left(V_{+} \leftrightarrow I_{+}, V_{-} \leftrightarrow I_{-}\right)$on the same device and measuring the direct SHE, as detailed in Supplemental Material 42 . It is well-known that the AHE occurs in FMs as a result of the breaking of time reversal symmetry. However, the Onsager reciprocal relation holds for the SHE in $\mathrm{FMs}^{43}$ because the total number of spin-up and spin-down electrons is always kept constant.

By using the spin transport model proposed by Takahashi and Maekawa ${ }^{44}$, the spin Hall resistivity $-\rho_{x y}^{\mathrm{SHE}}$ can be estimated as follows:

$$
-\rho_{x y}^{\mathrm{SHE}}=\rho_{y x}^{\mathrm{SHE}}=\theta_{\mathrm{SHE}} \rho_{x x}=\frac{w_{\mathrm{M}}}{x}\left(\frac{I_{\mathrm{C}}}{\bar{I}_{\mathrm{S}}}\right) \Delta R_{\mathrm{ISHE}}
$$

where $x$ is the shunting factor and $\bar{I}_{\mathrm{S}}$ is the effective spin current absorbed into the FM middle wire. When $\bar{I}_{\mathrm{S}}$ is converted into charge current in the middle wire, a part of the charge current is shunted by the $\mathrm{Cu}$ bridge on the middle FM wire. The shunting factor $x$ has been calculated with a finite elements method using SpinFlow $3 \mathrm{D}^{16,19}$. $\bar{I}_{\mathrm{S}}$ can be determined from nonlocal spin valve measurements with and without the middle wire ${ }^{42}$. $\bar{I}_{\mathrm{S}}$ is also related to the spin diffusion length of the middle FM wire.

We next measured the AHE with a Hall bar pattern, prepared at the same time as the SHE device. By applying an out-of-plane magnetic field and flowing $I_{\mathrm{C}}$ in the longitudinal direction of the Hall bar, a transverse voltage drop $V_{\mathrm{AHE}}\left(=V_{+}-V_{-}\right)$is detected, as sketched in the inset of Fig. 1(d). Figure 1(d) shows a typical $R_{\mathrm{AHE}}=V_{\mathrm{AHE}} / I_{\mathrm{C}}$ vs $H$ curve for Py at $10 \mathrm{~K}$. Although there are two backgrounds, namely normal Hall resistance and planar Hall resistance in between $\pm 10 \mathrm{kOe} \underline{45}$, a clear positive AHE signal $\Delta R_{\mathrm{AHE}}$ can be extracted. From $\Delta R_{\mathrm{AHE}}$, we obtain the anomalous Hall resistivity 

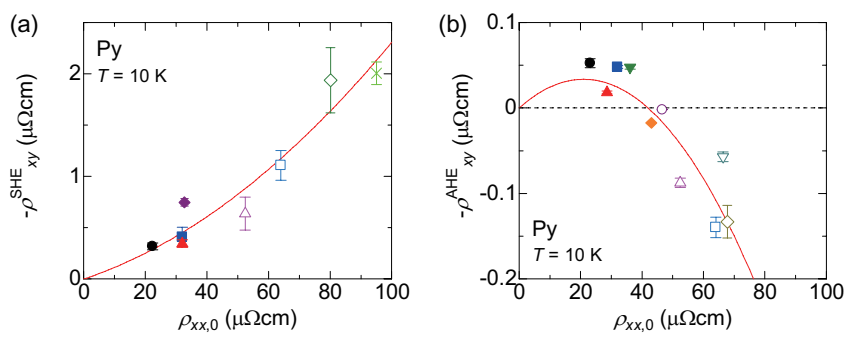

FIG. 2: (a) Spin Hall resistivity $-\rho_{x y}^{\mathrm{SHE}}$ and (b) anomalous Hall resistivity $-\rho_{x y}^{\mathrm{AHE}}$ in $\mathrm{Py}$ as a function of $\rho_{x x, 0}$ at $10 \mathrm{~K}$. The solid lines are the best fits with Eq. (2). The same symbol is used in (a) and (b) if the Py deposition is done at the same time for the SHE and AHE samples. The dotted line in (b) indicates $-\rho_{x y}^{\mathrm{AHE}}=0$.

defined as,

$$
-\rho_{x y}^{\mathrm{AHE}}=\rho_{y x}^{\mathrm{AHE}}=\theta_{\mathrm{AHE}} \rho_{x x}=t \Delta R_{\mathrm{AHE}},
$$

where $\theta_{\mathrm{AHE}}$ is the anomalous Hall angle and $t$ is the thickness of the Hall bar.

By plotting $-\rho_{x y}^{\mathrm{SHE}}$ and $-\rho_{x y}^{\mathrm{AHE}}$, obtained with Eqs. (1) and (2), as a function of $\rho_{x x}$, the detailed mechanisms can be addressed as shown in previous works 16,46,47. For this purpose, the recent scaling equation proposed by Hou et al $\stackrel{46}{\underline{40}}$ is useful:

$$
\begin{aligned}
-\rho_{x y}^{\mathrm{H}}=\alpha_{\mathrm{ss}}^{\mathrm{H}} \rho_{x x, 0} & +\beta_{0}^{\mathrm{H}}\left(\rho_{x x, 0}\right)^{2} \\
& +\gamma^{\mathrm{H}} \rho_{x x, 0} \rho_{x x, T}+\beta_{1}^{\mathrm{H}}\left(\rho_{x x, T}\right)^{2},
\end{aligned}
$$

where $\mathrm{H}$ refers to the SHE or AHE, $\rho_{x x, 0}$ is the residual resistivity at low temperature (in the present case, at $10 \mathrm{~K}), \rho_{x x, T}\left(=\rho_{x x}-\rho_{x x, 0}\right)$ is the resistivity induced by phonons and $\alpha_{\mathrm{ss}}^{\mathrm{H}}$ is the skew scattering angle due to impurities or grain boundaries. As detailed in Ref. 46, the side-jump terms due to static (impurities or grain boundaries) and dynamic (phonons) scattering sources as well as the intrinsic contribution originating from the band structure $\underline{48-54}$ are entangled in $\beta_{0}^{\mathrm{H}}, \gamma^{\mathrm{H}}$, and $\beta_{1}^{\mathrm{H}}$ in a complex manner. Nevertheless, as discussed in Ref.46, the effect of the intrinsic Berry curvature is most strongly reflected in the $\beta_{1}^{\mathrm{H}}$ term.

Firstly, to simplify Eq. (3), we focus on the low temperature part where the phonon contribution is negligible and consider the case of Py. By substituting $\rho_{x x, T}=0$ in Eq. (3), a simplified equation can be obtained:

$$
-\rho_{x y}^{\mathrm{H}}=\alpha_{\mathrm{ss}}^{\mathrm{H}} \rho_{x x, 0}+\beta_{0}^{\mathrm{H}}\left(\rho_{x x, 0}\right)^{2} .
$$

In order to determine $\alpha_{\mathrm{ss}}^{\mathrm{H}}$ and $\beta_{0}^{\mathrm{H}}$, the SHE and AHE of Py have to be measured in a wide $\rho_{x x, 0}$ range. For this purpose, we changed the thickness of the Py wire (from 5 to $30 \mathrm{~nm}$ ) and also the deposition rate (from $0.04 \mathrm{~nm} / \mathrm{s}$ to $0.08 \mathrm{~nm} / \mathrm{s}$ ), as already demonstrated in our previous work for $\mathrm{Pt} \underline{16}$. Figures 2 (a) and 2(b) show $-\rho_{x y}^{\mathrm{SHE}}$ and $-\rho_{x y}^{\mathrm{AHE}}$ of Py at $10 \mathrm{~K}$ as a function of $\rho_{x x, 0}$,
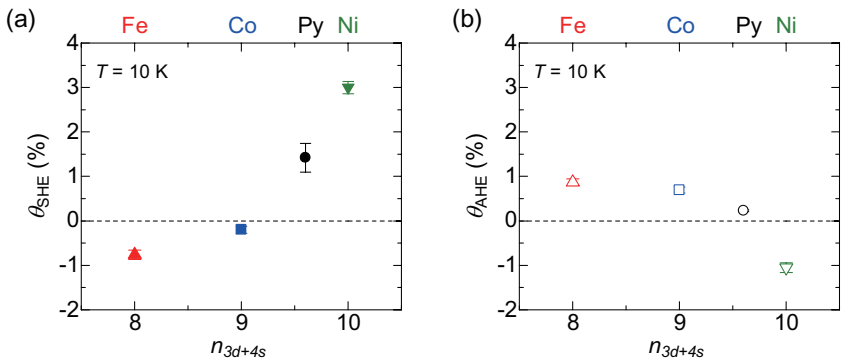

FIG. 3: (a) Spin Hall angle $\theta_{\mathrm{SHE}}$ and (b) anomalous Hall angle $\theta_{\mathrm{AHE}}$ measured at $10 \mathrm{~K}$ as a function of the number of electrons in the outermost shell. The thickness of the the $3 d \mathrm{FMs}$ is $20 \mathrm{~nm}$. The dotted lines in (a) and (b) indicate $\theta_{\mathrm{H}}=0$.

respectively. $-\rho_{x y}^{\mathrm{SHE}}$ increases with increasing $\rho_{x x, 0}$, while $-\rho_{x y}^{\mathrm{AHE}}$ decreases with $\rho_{x x, 0}$. By fitting $-\rho_{x y}^{\mathrm{SHE}}$ and $-\rho_{x y}^{\mathrm{AHE}}$ with Eq. (4), the skew scattering term $\alpha_{\mathrm{ss}}^{\mathrm{H}}$ and the combination of the side-jump and intrinsic contributions $\beta_{0}^{\mathrm{H}}$ can be obtained as follows: $\alpha_{\mathrm{ss}}^{\mathrm{SHE}}=1.0 \pm 0.4 \%$, $\alpha_{\mathrm{ss}}^{\mathrm{AHE}}=0.32 \pm 0.1 \%, \beta_{0}^{\mathrm{SHE}}=131 \pm 60 \Omega^{-1} \cdot \mathrm{cm}^{-1}$ and $\beta_{0}^{\mathrm{ASH}}=-76 \pm 20 \Omega^{-1} \cdot \mathrm{cm}^{-1} \cdot \alpha_{\mathrm{ss}}^{\mathrm{AHE}}$ and $\beta_{0}^{\mathrm{AHE}}$ are in good agreement with previous reports 45.55 .

Interestingly, the ratio of the AHE and SHE in Py for the skew scattering contribution, $\alpha_{\mathrm{ss}}^{\mathrm{AHE}} / \alpha_{\mathrm{SS}}^{\mathrm{SHE}}=0.32$, is a reasonable value for the spin polarization $p$ of $\mathrm{Py} \underline{18,56}$. In other words, for the skew scattering, the relation between the AHE and SHE can be expressed as

$$
\rho_{x y}^{\mathrm{AHE}}=p \rho_{x y}^{\mathrm{SHE}} .
$$

This can be understood intuitively as follows. In FMs, incident spin-up and spin-down electrons are deflected to the transverse opposite directions, as illustrated in Fig. 1(a). Since the number of spin-up electrons (3 in the sketch) is larger than that of spin-down electrons (2 in the sketch), there is a finite charge accumulation (the difference of the deflected electrons, i.e., 1 in the sketch) along the transverse direction with respect to the incident current direction, which can be detected as an anomalous Hall voltage. On the other hand, the spin accumulation is proportional to the difference of spin directions ( 5 in the sketch), which can be detected as a spin Hall voltage. Thus, the ratio of the AHE and SHE is indeed the spin polarization $(p=(3-2) /(3+2)=0.2$ in the sketch of Fig. 1(a)).

In fact, this simple picture can be applied for diffusive scattering systems such as Py. As we detail in Ref. 42, Eq. (5) can be derived in Mott's two current model 157 under the specific assumption $\frac{\rho_{x y}^{\uparrow}}{\rho_{x x}^{\uparrow}}=-\frac{\rho_{x y}^{\downarrow}}{\rho_{x x}^{\downarrow}}$. Here $\rho_{i j}^{\uparrow}$ and $\rho_{i j}^{\downarrow}$ are the spin-up and spin-down resistivity tensor elements. Py is a random alloy composed of $\mathrm{Ni}$ and $\mathrm{Fe}$. The anisotropy on the Fermi surface should be suppressed and lead to more isotropic scattering properties. Thus, the Hall angle is essentially a spin-independent property 
TABLE I: The coefficients $\beta_{1}^{\mathrm{H}}$ and $\gamma^{\mathrm{H}}$ extracted from the fittings with Eq. (3) for each FM. For comparison, we also show the coefficient of the quadratic term of the AHE from previous works (Refs. $45,49,52,54$ ) in the table.

\begin{tabular}{|c|c|c|c|c|c|}
\hline FM & $\begin{array}{c}\beta_{1}^{\text {SHE }} \\
\left(\times 10^{3} \Omega^{-1} \mathrm{~cm}^{-1}\right) \\
\end{array}$ & $\begin{array}{c}\beta_{1}^{\mathrm{AHE}} \\
\left(\times 10^{3} \Omega^{-1} \mathrm{~cm}^{-1}\right) \\
\end{array}$ & $\begin{array}{c}\beta_{1}^{\mathrm{AHE}} \text { or } b^{55} \text { in literature } \\
\left(\times 10^{3} \Omega^{-1} \mathrm{~cm}^{-1}\right)\end{array}$ & $\begin{array}{c}\gamma^{\mathrm{SHE}} \\
\left(\times 10^{3} \Omega^{-1} \mathrm{~cm}^{-1}\right)\end{array}$ & $\begin{array}{c}\gamma^{\mathrm{AHE}} \\
\left(\times 10^{3} \Omega^{-1} \mathrm{~cm}^{-1}\right)\end{array}$ \\
\hline $\mathrm{Fe}$ & $4.9 \pm 0.2$ & $0.89 \pm 0.04$ & $1.1^{45}, 0.82^{53}$ & $-1.1 \pm 0.1$ & $1.50 \pm 0.03$ \\
\hline Co & $-8.3 \pm 0.5$ & $0.34 \pm 0.03$ & $0.2^{54}, 0.73^{49}$ & $0.04 \pm 0.24$ & $0.97 \pm 0.02$ \\
\hline $\mathrm{Py}$ & $-10.1 \pm 0.3$ & $-0.056 \pm 0.015$ & $-0.05^{45}$ & $0.57 \pm 0.14$ & $-0.002 \pm 0.009$ \\
\hline $\mathrm{Ni}$ & $-17.1 \pm 0.5$ & $-0.14 \pm 0.11$ & $-(0.5 \sim 1.0)^{52}$ & $5.9 \pm 0.4$ & $-0.89 \pm 0.09$ \\
\hline
\end{tabular}

(a)

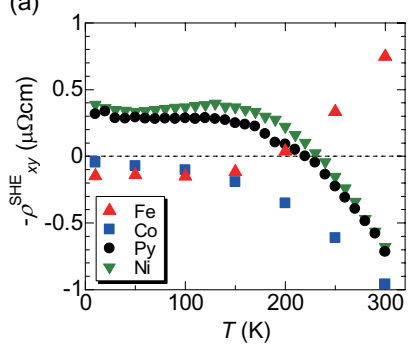

(b)

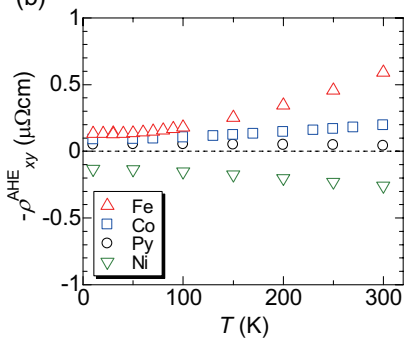

FIG. 4: The temperature dependence of (a) spin Hall resistivity $-\rho_{x y}^{\mathrm{SHE}}$ and (b) anomalous Hall resistivity $-\rho_{x y}^{\mathrm{AHE}}$ in four FMs (Py, Fe, Co and Ni). The thickness of the four FMs is $20 \mathrm{~nm}$ both for the SHE and AHE measurements.

averaged over all the contributing states. This supports the finding that the simplified relation holds for the skew scattering in $\mathrm{Py}$.

\section{B. SHE and AHE in other $3 d$ FMs}

However, such a simple picture does not work for the other $3 d$ FMs. The electronic states can be quite anisotropic because of the complicated band structure of $3 d$ FMs. Those states should show distinct effective spin-orbit couplings for spin-up and spin-down electrons. Thus, the above specific assumption can break down. We show $\theta_{\mathrm{SHE}}$ and $\theta_{\mathrm{AHE}}$ at $T=10 \mathrm{~K}$ for the $3 d \mathrm{FMs}$ in Figs. 3(a) and 3(b), respectively. As in the case of the intrinsic SHEs in $4 d$ and $5 d$ transition metals $13,26,27, \theta_{\mathrm{SHE}}$ is expected to change the sign from negative to positive with increasing the number of electrons in the outer shell ${ }^{32}$. Such a tendency can be seen clearly in $\theta_{\mathrm{SHE}}$ of the $3 d$ FMs in Fig. 3(a). However, the sign of $\theta_{\mathrm{SHE}}$ is opposite to that of $\theta_{\mathrm{AHE}}$ for Fe, Co, and Ni. Even in the case of Py, $\theta_{\mathrm{AHE}}$ is negative when $\rho_{x x, 0}$ is more than $40 \mu \Omega \cdot \mathrm{cm}$, as shown in Fig. 2(b). This obviously shows that Eq. (5) is not general and the detailed band structure of the electron orbitals has to be taken into account, as mentioned above.

So far, we have focused on the low temperature parts of the SHE and AHE. To address the effect of dynamic disorders, we next discuss the temperature dependences of the SHE and AHE in Figs. 4(a) and 4(b), respec- tively. The temperature dependence of the SHE is much stronger than that of the AHE. For Fe, Py, and Ni, the sign of $-\rho_{x y}^{\mathrm{SHE}}$ is changed at $200-250 \mathrm{~K}$, while such a sign change cannot be seen for $-\rho_{x y}^{\mathrm{AHE}}$. To specify the reason for such temperature dependences, we have fitted both $-\rho_{x y}^{\mathrm{SHE}}$ and $-\rho_{x y}^{\mathrm{AHE}}$ as a function of $\rho_{x x, T}$ with Eq. (3) as shown in Fig. 5] and obtained $\beta_{1}^{\mathrm{H}}$ and $\gamma^{\mathrm{H}}$ as the quadratic and linear terms in Eq. (3), respectively (see Table I). For example, $\beta_{1}^{\mathrm{SHE}}$ of Py is more than two orders of magnitude larger than $\beta_{1}^{\mathrm{AHE}}$. Even for Py, the relations between the SHE and AHE for $\beta_{1}^{\mathrm{H}}$ and $\gamma^{\mathrm{H}}$ are not as simple as the skew scattering term.

A similar tendency can be seen for the other $3 d$ FMs. For $\mathrm{Fe}, \mathrm{Co}$, and $\mathrm{Ni}, \beta_{1}^{\mathrm{SHE}}$ is one or two orders of magnitude larger than $\beta_{1}^{\mathrm{AHE}}$, as shown in Table [1. Note that $\beta_{1}^{\mathrm{AHE}}$ values in the present work are in good agreement with previous experiments (see Table I) and tightbinding calculations 58 . On the other hand, $\left|\beta_{1}^{\mathrm{SHE}}\right|$ of the $3 d$ FMs ranges between 4.9 and $17 \times 10^{3} \Omega^{-1} \cdot \mathrm{cm}^{-1}$, which is larger than that of a typical SHE material, $\mathrm{Pt}$ $\left(1.6 \times 10^{3} \Omega^{-1} \cdot \mathrm{cm}^{-1}\right)^{13,16}$. The relation between $\gamma^{\mathrm{SHE}}$ and $\gamma^{\text {AHE }}$ strongly varies with the $3 d$ FMs (see Table $\llbracket$ ).

Much larger $\beta_{1}^{\mathrm{SHE}}$ values than $\beta_{1}^{\mathrm{AHE}}$ ones would originate from the stronger temperature dependence of the $\mathrm{SHE}$ in $3 d \mathrm{FMs}$. At the moment, we do not have a conclusive picture for the origin of this dependence. In general, the spin transport can be mediated not only by conduction electrons but also by magnons in $\mathrm{FMs}^{59,60}$. One possible scenario is the contribution of electron-magnon interactions in $3 d$ FMs. The electron-magnon interactions would induce additional spin-flip processes. We note that such spin-flip processes are equivalent in magnitude for up-to-down and down-to-up spin channels even in ferromagnetic systems 61 . In such a situation, some asymmetric scatterings which are spin-dependent would contribute only to the SHE but not to the AHE, and thus would be associated with the fact that the strong temperature dependence is not present in the AHE of the $3 d$ FMs or the SHE of nonmagnetic metals. Interestingly, a recent theoretical report claims that magnon spin current can be significant around room temperature in $3 d$ $\mathrm{FMs}^{62}$, which might be related to our case. However, there are some open questions: how large the asymmetric scatterings are quantitatively and whether any other mechanisms contribute to the observed spin Hall resistivity or not. These would be addressed in future. 

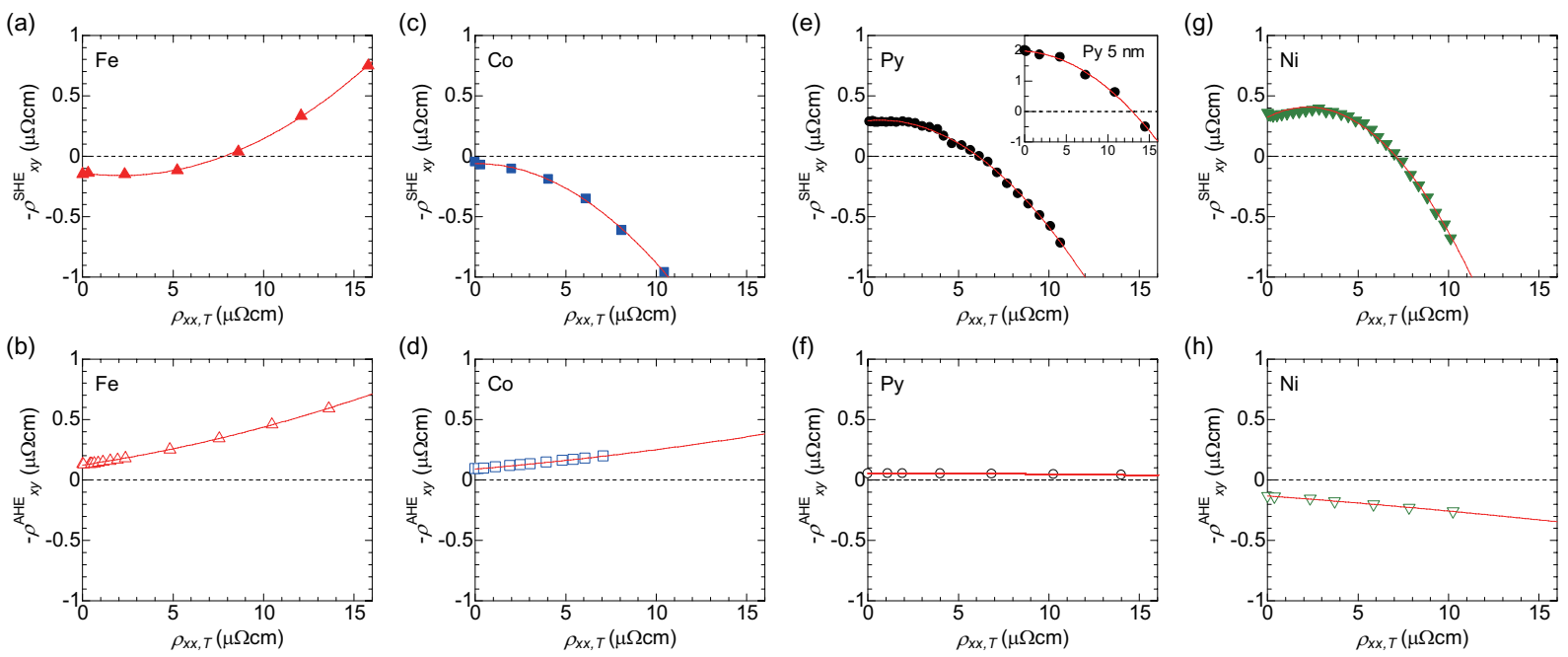

FIG. 5: Spin Hall resistivity $-\rho_{x y}^{\mathrm{SHE}}$ and anomalous Hall resistivity $-\rho_{x y}^{\mathrm{AHE}}$ in four FMs (Fe, Co, Py, and Ni) at finite temperatures. $-\rho_{x y}^{\mathrm{SHE}}$ as a function of $\rho_{x x, T}$ in (a) Fe, (c) Co, (e) Py, and (g) Ni. Anomalous Hall resistivity $-\rho_{x y}^{\mathrm{AHE}}$ as a function of $\rho_{x x, T}$ in (b) Fe, (d) Co, (f) Py, and (h) Ni. $\rho_{x x, T}$ varies by changing temperature from $10 \mathrm{~K}$ to $300 \mathrm{~K}$. The solid lines are the best fits of the data to Eq. (3). The thickness of the four FMs is $20 \mathrm{~nm}$ both for SHE and AHE measurements except for the inset in (e). The inset in (e) shows $-\rho_{x y}^{\mathrm{SHE}}$ of $5 \mathrm{~nm}$ thick Py wire. For the fitting with Eq. (3)), the same parameter $\beta_{1}^{\mathrm{SHE}}=-10.1\left(\times 10^{3} \Omega^{-1} \cdot \mathrm{cm}^{-1}\right)$ was used both for $20 \mathrm{~nm}$ and $5 \mathrm{~nm}$ thick Py wires.

\section{SUMMARY}

In conclusion, we experimentally investigated the relation between the SHE and AHE in four $3 d \mathrm{FMs}[\mathrm{Fe}$, $\mathrm{Co}, \mathrm{Py}\left(\mathrm{Ni}_{81} \mathrm{Fe}_{19}\right)$, and $\left.\mathrm{Ni}\right]$. In a typical ferromagnetic alloy, Py, the skew scattering contribution of the AHE is related to that of the SHE via the spin polarization of Py, as can be understood intuitively. However, this relation does not hold for other mechanisms. This fact is highlighted by the temperature dependence of the SHE and AHE. For all the $3 d \mathrm{FMs}$, one of the intrinsic mechanism terms $\beta_{1}^{\mathrm{SHE}}$ is much larger than $\beta_{1}^{\mathrm{AHE}}$. Asymmetric spindependent scatterings in the spin-flip processes induced by the electron-magnon interactions would be a possible explanation for the strong temperature dependence of the SHE in contrast to the AHE or even the SHE in nonmagnetic metals.

\section{Acknowledgments}

We thank S. Maekawa and B. Gu for fruitful discussions. This work is supported by the Japanese Grant-
in-Aid for Scientific Research on Innovative Area, "Nano Spin Conversion Science" (Grant No. JP26103002), and by the Spanish MINECO under the Maria de Maeztu Units of Excellence Programme (MDM-2016-0618) and under Projects No. MAT2015-65159-R and MAT201782071-ERC. Y. Omori acknowledges financial support from Japan Society for the Promotion of Science (JSPS) through "Research program for Young Scientists" and "Program for Leading Graduate Schools (MERIT)". M.G. acknowledges financial support from the Leverhulme Trust via an Early Career Research Fellowship (ECF-2013-538). E.S thanks the Spanish Ministry of Education, Culture and Sport for a Ph.D. fellowship (Grant No. FPU14/03102).
* These authors contributed equally to this work.

† Electronic address: niimi@phys.sci.osaka-u.ac.jp

‡ Electronic address: yotani@issp.u-tokyo.ac.jp

1 A. Hoffmann, IEEE Trans. Magn. 49, 5172 (2013).

2 J. Sinova, S. O. Valenzuela, J. Wunderlich, C. H. Back, and T. Jungwirth, Rev. Mod. Phys. 87, 1213 (2015).
3 Y. Niimi and Y. Otani, Rep. Prog. Phys. 78, 124501 (2015).

4 M. I. Dyakonov and V. I. Perel, Phys. Lett. A 35, 459 (1971).

5 J. E. Hirsch, Phys. Rev. Lett. 83, 1834 (1999).

${ }^{6}$ Y. K. Kato, R. S. Myers, A. C. Gossard and D. D. 
Awschalom, Science 306, 1910 (2004).

7 J. Wunderlich, B. Kaestner, J. Sinova, and T. Jungwirth, Phys. Rev. Lett. 94, 047204 (2005).

8 E. Saitoh, M. Ueda, H. Miyajima, and G. Tatara, Appl. Phys. Lett. 88, 182509 (2006).

${ }^{9}$ K. Ando, S. Takahashi, K. Harii, K. Sasage, J. Ieda, S. Maekawa, and E. Saitoh, Phys. Rev. Lett. 101, 036601 (2008).

10 O. Mosendz, J. E. Pearson, F. Y. Fradin, G. E. W. Bauer, S. D. Bader, A. Hoffmann, Phys. Rev. Lett. 104, 046601 (2010).

11 O. Mosendz, V. Vlaminck, J. E. Pearson, F. Y. Fradin, G. E. W. Bauer, S. D. Bader, and A. Hoffmann, Phys. Rev. B 82, 214403 (2010).

12 L. Liu, T. Moriyama, D. C. Ralph, and R. A. Buhrman, Phys. Rev. Lett. 106, 036601 (2011).

13 M. Morota, Y. Niimi, K. Ohnishi, D. H. Wei, T. Tanaka, H. Kontani, T. Kimura, and Y. Otani, Phys. Rev. B 83, 174405 (2011).

${ }^{14}$ K. Kondou, H. Sukegawa, S. Mitani, K. Tsukagoshi, and S. Kasai, Appl. Phys. Express 5, 073002 (2012).

15 M. Isasa, E. Villamor, L. E. Hueso, M. Gradhand and F. Casanova, Phys. Rev. B 91, 024402 (2015).

16 E. Sagasta, Y. Omori, M. Isasa, M. Gradhand, L. E. Hueso, Y. Niimi, Y. Otani, and F. Casanova, Phys. Rev. B 94, 060412(R) (2016).

17 B. Gu, I. Sugai, T. Ziman, G.Y. Guo, N. Nagaosa, T. Seki, K. Takanashi, and S. Maekawa, Phys. Rev. Lett 105, 216401 (2010).

18 Y. Niimi, M. Morota, D. H. Wei, C. Deranlot, M. Basletic, A. Hamzic, A. Fert, and Y. Otani, Phys. Rev. Lett. 106, 126601 (2011).

19 Y. Niimi, Y. Kawanishi, D. H. Wei, C. Deranlot, H. X. Yang, M. Chshiev, T. Valet, A. Fert, and Y. Otani, Phys. Rev. Lett. 109, 156602 (2012).

20 P. Laczkowski, J.-C. Rojas-Sanchez, W. Savero-Torres, H. Jaffres, N. Reyren, C. Deranlot, L. Notin, C. Beigne, A. Marty, J.-P. Attane, L. Vila, J.-M. George, and A. Fert, Appl. Phys. Lett. 104, 142403 (2014).

${ }^{21}$ K. Fujiwara, Y. Fukuma, J. Matsuno, H. Idzuchi, Y. Niimi, Y. Otani, and H. Takagi, Nat. Commun. 4, 2893 (2013).

${ }^{22}$ K. Ando, S. Watanabe, S. Mooser, E. Saitoh, and H. Sirringhaus, Nat. Mater. 12, 622 (2013).

23 M. Gradhand, D. V. Fedorov, P. Zahn, and I. Mertig, Phys. Rev. Lett. 104, 186403 (2010).

24 S. Lowitzer, M. Gradhand, D. Ködderitzsch, D. V. Fedorov, I. Mertig, and H. Ebert, Phys. Rev. Lett. 106, 056601 (2011).

25 A. Fert and P. M. Levy, Phys. Rev. Lett. 106, 157208 (2011).

26 G. Y. Guo, S. Murakami, T.-W. Chen, and N. Nagaosa, Phys. Rev. Lett. 100, 096401 (2008).

27 T. Tanaka, H. Kontani, M. Naito, T. Naito, D. S. Hirashima, K. Yamada, and J. Inoue, Phys. Rev. B 77, 165117 (2008).

28 N. Nagaosa, J. Sinova, S. Onoda, A. H. MacDonald, and N. P. Ong, Rev. Mod. Phys. 82, 1539 (2010).

29 B. Zimmermann, K. Chadova, D. Ködderitzsch, S. Blügel, H. Ebert, D. V. Fedorov, N. H. Long, P. Mavropoulos, I. Mertig, Y. Mokrousov, and M. Gradhand, Phys. Rev. B 90, 220403(R) (2014).

30 B. F. Miao, S. Y. Huang, D. Qu, and C. L. Chien, Phys. Rev. Lett. 111, 066602 (2013)

31 A. Tsukahara, Y. Ando, Y. Kitamura, H. Emoto, E.
Shikoh, M. P. Delmo, T. Shinjo, and M. Shiraishi, Phys. Rev. B 89, 235317 (2014).

32 C. Du, H. Wang, F. Yang, and P. C. Hammel, Phys. Rev. B 90, 140407(R) (2014).

33 K. S. Das, W. Y. Schoemaker, B. J. van Wees, and I. J. Vera-Marun, Phys. Rev. B 96, 220408(R) (2017).

${ }^{34}$ K. S. Das, J. Liu, B. J. van Wees, and I. J. Vera-Marun, Nano Lett. 18, 5633 (2018).

35 J. D. Gibbons, D. MacNeill, R. A. Buhrman, and D. C. Ralph, Phys. Rev. Applied 9, 064033 (2018).

36 S. Iihama, T. Taniguchi, K. Yakushiji, A. Fukushima, Y. Shiota, S. Tsunegi, R. Hiramatsu, S. Yuasa, Y. Suzuki and H. Kubota, Nat. Electronics 1, 120 (2018).

37 C. Qin, S. Chen, Y. Cai, F. Kandaz, and Y. Ji, Phys. Rev. B 96, 134418 (2017)

38 T. Taniguchi, J. Grollier and M. D. Stiles, Phys. Rev. Applied 3, 044001 (2015).

39 M. Seemann, D. Koedderitzsch, S. Wimmer, and H. Ebert, Phys. Rev. B 92, 155138 (2015).

40 Within the semiclassical picture, the anomalous Hall and spin Hall conductivities arising from scatterings are given by $\sigma_{x y}^{\mathrm{AHE}}=-\frac{\mathrm{e}^{2}}{\hbar} \frac{1}{(2 \pi)^{3}} \int_{\mathrm{FS}} \frac{v_{x}(\mathbf{k}) \lambda_{y}(\mathbf{k})}{|\mathbf{v}(\mathbf{k})|} \mathrm{d} S$ and $\sigma_{x y}^{\mathrm{SHE}}=$ $-\frac{\mathrm{e}^{2}}{\hbar} \frac{1}{(2 \pi)^{3}} \int_{\mathrm{FS}} s_{z}(\mathbf{k}) \frac{v_{x}(\mathbf{k}) \lambda_{y}(\mathbf{k})}{|\mathbf{v}(\mathbf{k})|} \mathrm{d} S$, respectively. Here $v_{i}(\mathbf{k})$ are the Fermi velocities, $\lambda_{i}(\mathbf{k})$ is the mean free path, $s_{z}(\mathbf{k})$ is the spin polarization and we integrate over the Fermi surface (FS). In the limit of diffusive transport with an isotropic spin polarization $s_{z}(\mathbf{k})=p$, we can take $p$ in front of the integral and we find $\rho_{x y}^{\mathrm{AHE}}=p \rho_{x y}^{\mathrm{SHE}}$.

41 J. Smit, Physica 24, 39 (1958).

42 See Supplemental Material at https://... for details of the evaluation of the spin diffusion length of the middle wire and $\bar{I}_{\mathrm{S}}$, the Onsager reciprocity in Py, and the relation between SHE and AHE using the simplified model.

${ }^{43}$ P. Jacquod, R. S. Whitney, J. Meair, and M. Büttiker, Phys. Rev. B 86, 155118 (2012).

44 S. Takahashi and S. Maekawa, Phys. Rev. B 67, 052409 (2003); Sci. Tech. Adv. Mater. 9, 014105 (2008).

45 Y. Q. Zhang, N. Y. Sun, R. Shan, J. W. Zhang, S. M. Zhou, Z. Shi, and G. Y. Guo, J. Appl. Phys. 114, 163714 (2013).

46 D. Hou, G. Su, Y. Tian, X. Jin, S. A. Yang, and Q. Niu, Phys. Rev. Lett. 114, 217203 (2015).

47 Y. Tian, L. Ye, and X. Jin, Phys. Rev. Lett. 103, 087206 (2009).

48 S. A. Yang, H. Pan, Y. Yao, and Q. Niu, Phys. Rev. B 83, $125122(2011)$

49 D. Hou, Y. Li, D.Wei, D. Tian, L.Wu, and X. Jin, J. Phys.: Condens. Matter 24, 482001 (2012).

50 L. J. Zhu, D. Pan, and J. H. Zhao, Phys. Rev. B 89, 220406 (2014).

51 J. Xu, L. Wu, Y. Li, D. Tian, K. Zhu, X. Gong and X. Jin, Sci. Bull. 60, 1261 (2015).

52 L. Ye, Y. Tian, X. Jin, and D. Xiao, Phys. Rev. B 85, 220403(R) (2012).

${ }^{53}$ L. Wu, Y. Li, J. Xu, D. Hou, and X. Jin, Phys. Rev. B 87, 155307 (2013).

54 J. Kötzler and W. Gil, Phys. Rev. B 72, 060412(R) (2005).

55 Strictly speaking, $\beta_{0}^{\mathrm{AHE}}$ and $\beta_{1}^{\mathrm{AHE}}$ are different from $b$ which is the coefficient of $\rho_{x x}^{2}$ in Ref. 45. In general, $b$ should be closer to $\beta_{1}^{\mathrm{AHE}}$, but in the case of $\mathrm{Py}$, both $\beta_{0}^{\mathrm{AHE}}$ and $\beta_{1}^{\mathrm{AHE}}$ are comparable to $b$.

56 E. Sagasta, Y. Omori, M. Isasa, Y. Otani, L. E. Hueso, and F. Casanova, Appl. Phys. Lett. 111, 082407 (2017). 
57 N. F. Mott, Adv. Phys. 13, 325 (1964).

58 T. Naito, D. S. Hirashima, and H. Kontani, Phys. Rev. B 81, 195111 (2010).

59 J. Cramer, F. Fuhrmann, U. Ritzmann, V. Gall, T. Niizeki, R. Ramos, Z. Qiu, D. Hou, T. Kikkawa, J. Sinova, U. Nowak, E. Saitoh, and M. Kläui, Nat. Commun. 9, 1089 (2018).

60 L. J. Cornelissen, J. Liu, R. A. Duine, J. Ben Youssef and B. J. van Wees, Nat. Phys. 11, 1022 (2015).

61 The scattering probability related to the spin-flip process is defined as $P_{k k^{\prime}}^{+-} \sim\left|\left\langle\Psi_{k^{\prime}}^{-}|\hat{T}| \Psi_{k}^{+}\right\rangle\right|^{2}$ where $\Psi_{k\left(k^{\prime}\right)}^{+(-)}$is the wave function before (after) the scattering with the wave number $k\left(k^{\prime}\right)$ and spin-up $+($ spin-down -$)$, and $\hat{T}$ is a matrix in spin space including spin-orbit coupling and magnetism. By defining $P_{k k^{\prime}}^{-+}$in an equivalent way and integrating $k$ and $k^{\prime}$, we find that on average it holds $\tau_{+-}^{-1}=\tau_{-+}^{-1}$, with $\tau^{-1}=\int \mathrm{dk} \int \mathrm{dk}^{\prime} P_{k k^{\prime}}$, even in the magnetic case.

62 Y. Cheng, K. Chen, and S. Zhang, Phys. Rev. B 96, 024449 (2017). 